\title{
Primary Resonance of van der Pol Oscillator under Fractional-Order Delayed Feedback and Forced Excitation
}

\author{
Jufeng Chen, Xianghong Li, Jianhua Tang, and Yafeng Liu \\ Department of Mathematics and Physics, Shijiazhuang Tiedao University, Shijiazhuang, China \\ Correspondence should be addressed to Xianghong Li; 1xhll601@163.com
}

Received 27 June 2017; Accepted 21 November 2017; Published 18 December 2017

Academic Editor: Matteo Aureli

Copyright (C) 2017 Jufeng Chen et al. This is an open access article distributed under the Creative Commons Attribution License, which permits unrestricted use, distribution, and reproduction in any medium, provided the original work is properly cited.

\begin{abstract}
The primary resonance of van der Pol oscillator under fractional-order delayed negative feedback and forced excitation is studied. Firstly, the approximate analytical solution is obtained based on the averaging method, and it could be found that the fractionalorder delayed feedback has not only the property of delayed velocity feedback but also that of delayed displacement feedback. Moreover, the amplitude-frequency equation for the steady-state solution is established, and its stability conditions are also obtained. Then, the results of the approximate analytical solution and numerical integration are compared and analyzed. The agreement between the two methods is very high, so that the correctness and accuracy of the approximate analytical solution are verified. Finally, the effects of all the parameters in the fractional-order delayed feedback on the amplitude-frequency curves are analyzed. It could be concluded that fractional-order delayed feedback has important influences on the dynamical behavior of van der Pol oscillator, which is very significant to the optimization and control of a similar system.
\end{abstract}

\section{Introduction}

As an important branch of mathematics, fractional calculus had been studied for more than 300 years. In recent years, it had attracted more attention in a lot of research fields, such as physics, chemistry, mechanics, biology, electromagnetics, materials science, and control engineering [1-11]. This was due to the fact that many features in time and space could be explained by the fractional-order calculus model, such as memory and nonlocality. At present, the forms of fractional-order differential systems can be classified into two categories. The first one is to simply add fractional-order derivative term into the original integerorder system, so as to establish a fractional-order system. For example, Shen et al. [12-16] studied several linear and nonlinear fractional-order oscillators by the averaging method or incremental harmonic balance method and found that the fractional-order derivatives had both damping and stiffness effects on the dynamical response in those oscillators. Chen et al. $[17,18]$ studied the response of some nonlinear fractional-order oscillator under Gaussian white noise excitation. Yang et al. $[19,20]$ investigated the stochastic response of nonlinear system with Caputo-type fractional derivative subject to Gaussian white noise. Xu et al. [21] proposed a new technique to deal with strongly nonlinear stochastic systems with fractional derivative damping and random harmonic excitation. The other one is that the classic integer-order derivatives in dynamical system are directly extended to the fractional-order ones, so that a fractionalorder differential system in state space is obtained. This kind of systems include fractional-order Lorenz, van der Pol, and Duffing system, and one could study the stability region, bifurcations, chaos, and its control [22]. For example, Li and Peng [23] found that chaos existed in Chen's system with a fractional order by utilizing the fractional calculus techniques. Ahmed et al. [24] proposed some Routh-Hurwitz stability conditions for some fractional-order systems. $\mathrm{Li}$ and $\mathrm{Wu}$ [25] studied the chaotic behaviors and the Hopf bifurcation in a new fractional-order hyperchaotic system based on the Lorenz system. Čermák and Nechvátal [26] discussed the stability conditions and chaotic behavior of the Lorenz system involving the Caputo fractional derivative with order between 0 and 1 .

Time delay is more common and inevitable in dynamical and control systems, and it could lead to the instability 
of the dynamical system and the damage to the control performance [27-29]. At present, some researches had been done on fractional-order and time delay systems. For example, Deng et al. [30] studied the stability of $n$-dimensional linear fractional differential equation with time delays. Shi and Wang [31] presented the BIBO stability criterion of a fractional-order delayed system. Babakhani et al. [9] studied the existence of solutions at the neighborhood of equilibrium for fractional-order delayed differential equations and the Hopf bifurcations.

In recent years, the research on fractional-order van der Pol oscillator had attracted more and more attention of the scholars. For example, Guo et al. [32, 33] studied the steadystate solution of the fractional van der Pol system with time delay via residue harmonic balance technique. Liu et al. [34] analyzed the asymptotic behaviors of the steady-state responses of a fractional van der Pol oscillator by homotopy analysis method and memory-free principle. Xie and Lin [35] investigated the asymptotic solution of the van der Pol oscillator with small fractional damping by using the method of two-scale expansion. Shen et al. [36] obtained the approximately analytical solution for the limit cycle of van der Pol oscillator with two kinds of fractional-order derivatives and analyzed its properties about amplitude and frequency. Wen et al. [37] investigated the influence of fractional-order delayed control on parameter-excited vibration for MathieuDuffing oscillator based on the switch of stability.

Different from the aforementioned references, the primary resonance of van der Pol oscillator under fractionalorder delayed feedback and forced excitation is analytically studied by averaging method in this paper. Particularly the effects of all the parameters in fractional-order delayed feedback on the primary resonance of the van der Pol system are studied, and the calculation process of the averaging method in fractional-order system is simplified. The paper is organized as follows. In Section 2 the approximate solution for primary resonance of van der Pol oscillator under fractional-order delayed feedback is obtained. The equivalent stiffness and damping coefficients denoted by the feedback gain, fractional order, time delay, and so on are defined. In Section 3 the stability condition of the steadystate solution is obtained. Then, the results of approximate analytical solution and numerical integration are compared by numerical simulation in Section 4 . Moreover, the effects of the parameters in the fractional-order delayed feedback on the amplitude-frequency equation are also given in this section. Finally, the main conclusions are made in Section 5.

\section{Approximate Analytical Solution of van der Pol Oscillator under Fractional-Order Delayed Feedback}

Van der Pol oscillator under forced excitation and fractionalorder delayed negative feedback is considered as follows:

$$
\begin{gathered}
m \ddot{x}(t)+k x(t)+\alpha_{1}\left[x^{2}(t)-1\right] \dot{x}(t) \\
=K_{1} D^{p}[x(t-\tau)]+F \cos (\omega t)
\end{gathered}
$$

where $m, k, \alpha_{1}, \tau, F$, and $\omega$ are the system mass, linear stiffness coefficient, nonlinear stiffness coefficient, time delay, excitation amplitude, and excitation frequency, respectively, $D^{p}[x(t-\tau)]$ is the $p$-order derivative of $x(t-\tau)$ to $t(0 \leq p \leq$ $1)$, and $K_{1}\left(K_{1}<0\right)$ is the fractional feedback gain. Here we adopt Caputo's definition [22]:

$$
D^{p}[x(t)]=\frac{1}{\Gamma(1-p)} \int_{0}^{t} \frac{x^{\prime}(u)}{(t-u)^{p}} \mathrm{~d} u,
$$

where $\Gamma(y)$ is Gamma function satisfying $\Gamma(y+1)=y \Gamma(y)$.

Introduce the following transformations:

$$
\begin{aligned}
\omega_{0} & =\sqrt{\frac{k}{m}}, \\
\varepsilon \alpha & =\frac{\alpha_{1}}{m}, \\
\varepsilon k_{1} & =\frac{K_{1}}{m}, \\
\varepsilon f & =\frac{F}{m} .
\end{aligned}
$$

Equation (1) becomes

$$
\begin{aligned}
\ddot{x}( & t)+\omega_{0}^{2} x(t)+\varepsilon \alpha\left[x^{2}(t)-1\right] \dot{x}(t) \\
= & \varepsilon k_{1} D^{p}[x(t-\tau)]+\varepsilon f \cos (\omega t),
\end{aligned}
$$

where $\omega_{0}$ is natural frequency and $\varepsilon$ is a small positive dimensionless parameter. We focus on the primary resonance by averaging method [12-15, 37], which means $\omega \approx \omega_{0}$. Hence, one could introduce $\omega^{2}=\omega_{0}^{2}+\varepsilon \sigma$ to illustrate the approximation degree, where $\sigma$ is the detuning factor.

Then, (4) can be written as

$$
\begin{aligned}
& \ddot{x}(t)+\omega^{2} x(t)=\varepsilon\left\{k_{1} D^{p}[x(t-\tau)]+f \cos (\omega t)\right. \\
& \left.+\sigma x(t)-\alpha\left[x^{2}(t)-1\right] \dot{x}(t)\right\} .
\end{aligned}
$$

Letting $\varphi=\omega t+\theta$, the solution of (5) can be assumed as

$$
\begin{aligned}
x(t) & =a \cos \varphi, \\
\dot{x}(t) & =-a \omega \sin \varphi, \\
x(t-\tau) & =a \cos (\varphi-\omega \tau),
\end{aligned}
$$

where the amplitude $a$ and the phase $\theta$ are slow-varying functions of time $t$.

Substituting (6a), (6b), and (6c) into (5), one could obtain

$$
\begin{aligned}
\dot{a} & =-\frac{1}{\omega}\left[P_{1}(a, \theta)+P_{2}(a, \theta, \tau)\right] \sin \varphi, \\
a \dot{\theta} & =-\frac{1}{\omega}\left[P_{1}(a, \theta)+P_{2}(a, \theta, \tau)\right] \cos \varphi,
\end{aligned}
$$

where

$$
\begin{array}{r}
P_{1}(a, \theta)=\varepsilon[f \cos (\varphi-\theta)+\sigma a \cos \varphi \\
\left.+\alpha \omega\left(a^{3}-a\right) \sin \varphi-\alpha \omega a^{3} \sin ^{3} \varphi\right] \\
P_{2}(a, \theta, \tau)=\varepsilon k_{1} D^{p}[a \cos (\varphi-\omega \tau)] .
\end{array}
$$


Applying the averaging method to (7a) and (7b) in time interval $[0, T]$, one could obtain

$$
\begin{aligned}
\dot{a} & =-\frac{1}{T \omega} \int_{0}^{T}\left[P_{1}(a, \theta)+P_{2}(a, \theta, \tau)\right] \sin \varphi \mathrm{d} \varphi, \\
a \dot{\theta} & =-\frac{1}{T \omega} \int_{0}^{T}\left[P_{1}(a, \theta)+P_{2}(a, \theta, \tau)\right] \cos \varphi \mathrm{d} \varphi .
\end{aligned}
$$

In the above equation, the time $T$ is selected as $T=l$ if $P_{1}(a, \theta)$ is a function with period $l$, or $T=\infty$ if $P_{2}(a, \theta, \tau)$ is an aperiodic one. One could obtain the simplified forms of the first part in (9a) and (9b)

$$
\begin{aligned}
\dot{a}_{1} & =-\frac{1}{2 \pi \omega} \int_{0}^{2 \pi} P_{1}(a, \theta) \sin \varphi \mathrm{d} \varphi \\
& =-\frac{\varepsilon f}{2 \omega} \sin \theta+\frac{\varepsilon \alpha a}{2}-\frac{\varepsilon \alpha a^{3}}{8}, \\
a \dot{\theta}_{1} & =-\frac{1}{2 \pi \omega} \int_{0}^{2 \pi} P_{1}(a, \theta) \cos \varphi \mathrm{d} \varphi \\
& =-\frac{\varepsilon f}{2 \omega} \cos \theta-\frac{\varepsilon \sigma a}{2 \omega} .
\end{aligned}
$$

In order to calculate the second part in (9a) and (9b), one could use the formula in literature [3]

$$
\begin{aligned}
& D^{p}[\cos (\mu t)]=\mu^{p} \cos \left(\mu t+\frac{p \pi}{2}\right), \\
& D^{p}[\sin (\mu t)]=\mu^{p} \sin \left(\mu t+\frac{p \pi}{2}\right) .
\end{aligned}
$$

Then, it yields

$$
\begin{aligned}
& D^{p}[\cos (\mu t+\sigma)]=\mu^{p} \cos \left(\mu t+\sigma+\frac{p \pi}{2}\right), \\
& D^{p}[\sin (\mu t+\sigma)]=\mu^{p} \sin \left(\mu t+\sigma+\frac{p \pi}{2}\right) .
\end{aligned}
$$

Hence,

$$
\begin{aligned}
\dot{a}_{2} & =-\lim _{T \rightarrow \infty} \frac{1}{T \omega} \int_{0}^{T} P_{2}(a, \theta, \tau) \sin (\omega t+\theta) \mathrm{d} t \\
& =\lim _{T \rightarrow \infty} \frac{-\varepsilon k_{1}}{T \omega} \int_{0}^{T} D^{p}[a \cos (\omega t+\theta-\omega \tau)] \sin (\omega t \\
& +\theta) \mathrm{d} t=-\frac{\varepsilon a k_{1}}{\omega} \lim _{T \rightarrow \infty} \frac{1}{T} \int_{0}^{T} \omega^{p} \\
& \cdot \cos \left(\omega t+\theta-\omega \tau+\frac{p \pi}{2}\right) \sin (\omega t+\theta) \mathrm{d} t \\
& =-\frac{\varepsilon a k_{1} \omega^{p-1}}{2} \lim _{T \rightarrow \infty} \frac{1}{T} \\
& \cdot \int_{0}^{T}\left[\sin \left(2 \omega t+2 \theta+\frac{p \pi}{2}-\omega \tau\right)\right. \\
& \left.-\sin \left(\frac{p \pi}{2}-\omega \tau\right)\right] \mathrm{d} t=\frac{\varepsilon a k_{1} \omega^{p-1}}{2} \sin \left(\frac{p \pi}{2}\right. \\
& -\omega \tau) .
\end{aligned}
$$

After a similar calculation, one could obtain

$$
\begin{aligned}
a \dot{\theta}_{2} & =-\lim _{T \rightarrow \infty} \frac{1}{T \omega} \int_{0}^{T} P_{2}(a, \theta, \tau) \cos (\omega t+\theta) \mathrm{d} t \\
& =\frac{-\varepsilon a k_{1} \omega^{p-1}}{2} \cos \left(\frac{p \pi}{2}-\omega \tau\right) .
\end{aligned}
$$

This calculation process is simpler than that in the literature [37].

Combining (10a) and (10b) with (13a) and (13b), one could obtain

$$
\begin{aligned}
\dot{a}= & -\frac{\varepsilon f}{2 \omega} \sin \theta+\frac{\varepsilon \alpha a}{2}-\frac{\varepsilon \alpha a^{3}}{8} \\
& +\frac{\varepsilon a k_{1} \omega^{p-1}}{2} \sin \left(\frac{p \pi}{2}-\omega \tau\right), \\
a \dot{\theta}= & -\frac{\varepsilon f}{2 \omega} \cos \theta-\frac{\varepsilon \sigma a}{2 \omega} \\
& -\frac{\varepsilon a k_{1} \omega^{p-1}}{2} \cos \left(\frac{p \pi}{2}-\omega \tau\right) .
\end{aligned}
$$

Substituting the parameters with the original ones, (14a) and (14b) could be written as

$$
\begin{aligned}
\dot{a}= & -\frac{F}{2 m \omega} \sin \theta+\frac{\alpha_{1} a}{2 m}-\frac{\alpha_{1} a^{3}}{8 m} \\
& +\frac{a K_{1} \omega^{p-1}}{2 m} \sin \left(\frac{p \pi}{2}-\omega \tau\right), \\
a \dot{\theta}= & -\frac{F}{2 m \omega} \cos \theta-\frac{\omega a}{2}+\frac{k a}{2 m \omega} \\
& -\frac{a K_{1} \omega^{p}}{2 m \omega} \cos \left(\frac{p \pi}{2}-\omega \tau\right) .
\end{aligned}
$$

Thus, one could get the approximate analytical solution of the system. Reorganizing (15a) and (15b), it yields

$$
\begin{aligned}
\dot{a} & =-\frac{F}{2 m \omega} \sin \theta-\frac{\alpha_{1} a^{3}}{8 m}-\frac{a}{2 m} C_{e}(p), \\
a \dot{\theta} & =-\frac{F}{2 m \omega} \cos \theta-\frac{\omega a}{2}+\frac{a}{2 m \omega} K_{e}(p),
\end{aligned}
$$

where

$$
\begin{aligned}
& C_{e}(p)=-\alpha_{1}-K_{1} \omega^{p-1} \sin \left(\frac{p \pi}{2}-\omega \tau\right), \\
& K_{e}(p)=k-K_{1} \omega^{p} \cos \left(\frac{p \pi}{2}-\omega \tau\right)
\end{aligned}
$$

are defined, respectively, as the equivalent damping coefficient and the equivalent stiffness coefficient.

From (16a), (16b), (17a), and (17b), we could conclude that the feedback gain $K_{1}$, fractional-order $p$, and time delay $\tau$ have important effects on $C_{e}(p)$ and $K_{e}(p)$. Since the feedback gain $K_{1}$ is linearly related to $C_{e}(p)$ and $K_{e}(p)$, it affects the response amplitude and resonance frequency in van der Pol 
oscillator simultaneously. When fractional-order $p \neq 0$, the fractional-order delayed feedback has the functions of both delayed displacement feedback and delayed velocity feedback. When $p \rightarrow 0$, fractional-order delayed feedback is almost equivalent to delayed displacement feedback. However, it is almost the same as delayed velocity feedback when $p \rightarrow 1$. Moreover, we could find that the amplitude and resonance frequency are affected periodically with the change of $\tau$.

\section{Amplitude-Frequency Equation and Stability Condition of the Approximate Solution}

Now we study the steady-state solution, which is more important and meaningful in vibration control. By putting $\dot{a}=0$ and $\dot{\theta}=0$ in (16a) and (16b), we could obtain

$$
\begin{array}{r}
4 F \sin \theta_{0}+a_{0}^{3} \alpha_{1} \omega+4 a_{0} \omega C_{e}(p)=0, \\
F \cos \theta_{0}+m \omega^{2} a_{0}-a_{0} K_{e}(p)=0,
\end{array}
$$

where $a_{0}$ and $\theta_{0}$ are the amplitude and phase of the steadystate solution, respectively.

Eliminating $\theta_{0}$ from (18a) and (18b), the amplitudefrequency equation is obtained as follows:

$$
\begin{aligned}
& \frac{a_{0}^{2}}{4}\left\{\left[\frac{\alpha_{1} a_{0}^{2}}{4}+C_{e}(p)\right]^{2}+\frac{1}{\omega^{2}}\left[K_{e}(p)-\omega^{2} m\right]^{2}\right\} \\
& \quad=\frac{F^{2}}{4 \omega^{2}} .
\end{aligned}
$$

For simplicity, one could define

$$
\rho \stackrel{\text { def }}{=} \frac{a_{0}^{2}}{4} \text {. }
$$

Then, another equivalent form of (19) can be written as

$$
\rho\left\{\left[\alpha_{1} \rho+C_{e}(p)\right]^{2}+\frac{1}{\omega^{2}}\left[K_{e}(p)-\omega^{2} m\right]^{2}\right\}=\frac{F^{2}}{4 \omega^{2}} .
$$

From (24), we could see that there may be one or three steadystate solutions in the case of primary resonance. Next, we study the stability of the steady-state solution. Letting $a=$ $a_{0}+\Delta a$ and $\theta=\theta_{0}+\Delta \theta$ and linearizing (16a) and (16b) at $\left(a_{0}, \theta_{0}\right)$, it yields

$$
\begin{aligned}
\frac{\mathrm{d} \Delta a}{\mathrm{~d} t} & =\left[-\frac{3 \alpha_{1} a_{0}^{2}}{8 m}-\frac{C_{e}(p)}{2 m}\right] \Delta a-\frac{F \cos \theta_{0}}{2 m \omega} \Delta \theta, \\
\frac{\mathrm{d} \Delta \theta}{\mathrm{d} t} & =\frac{F \cos \theta_{0}}{2 m a_{0}^{2} \omega} \Delta a+\frac{F \sin \theta_{0}}{2 m a_{0} \omega} \Delta \theta .
\end{aligned}
$$

Combined with (18a) and (18b), one could eliminate $\theta_{0}$ from (22a) and (22b) and get the characteristic equation as follows:

$$
\operatorname{det}\left(\begin{array}{cc}
A_{1}-\lambda & \frac{\omega a_{0}}{2}-\frac{a_{0} K_{e}(p)}{2 m \omega} \\
-\frac{\omega}{2 a_{0}}+\frac{K_{e}(p)}{2 m \omega a_{0}} & A_{2}-\lambda
\end{array}\right)=0 ;
$$

that is,

$$
\lambda^{2}-\left(A_{1}+A_{2}\right) \lambda+A_{1} A_{2}+\left[\frac{K_{e}(p)}{2 m \omega}-\frac{\omega}{2}\right]^{2}=0,
$$

where

$$
\begin{aligned}
& A_{1}=-\frac{3 \alpha_{1} a_{0}^{2}}{8 m}-\frac{C_{e}(p)}{2 m}=-\frac{3 \alpha_{1} \rho}{2 m}-\frac{C_{e}(p)}{2 m}, \\
& A_{2}=-\frac{\alpha_{1} a_{0}^{2}}{8 m}-\frac{C_{e}(p)}{2 m}=-\frac{\alpha_{1} \rho}{2 m}-\frac{C_{e}(p)}{2 m} .
\end{aligned}
$$

Based on the Hurwitz criterion, one could obtain the necessary and sufficient conditions for the stability of the steadystate solution as follows:

$$
\begin{aligned}
A_{1}+A_{2} & <0, \\
A_{1} A_{2}+\left[\frac{K_{e}(p)}{2 m \omega}-\frac{\omega}{2}\right]^{2} & >0 .
\end{aligned}
$$

Substituting (25) into (26), one could obtain the stability conditions as

$$
\begin{aligned}
& C_{e}(p)+2 \alpha_{1} \rho \\
& \quad=\alpha_{1}(2 \rho-1)-K_{1} \omega^{p-1} \sin \left(\frac{p \pi}{2}-\omega \tau\right)>0, \\
& {\left[C_{e}(p)+2 \alpha_{1} \rho\right]^{2}-\alpha_{1}^{2} \rho^{2}+\frac{1}{\omega^{2}}\left[K_{e}(p)-\omega^{2} m\right]^{2}} \\
& \quad>0 .
\end{aligned}
$$

\section{Numerical Simulation and the Effect of System Parameters}

4.1. Comparison between Approximate Analytical and Numerical Solution. In order to verify the correctness and precision of the approximate analytical solution, the numerical results of (1) are presented to compare the differences between the approximate analytical solutions and the numerical solutions. An illustrative example system is studied herein as defined by system parameters: $\alpha_{1}=1, m=4, k=10, K_{1}=-0.25$, $p=0.5$, and $F=1.4$.

Here we select time delay $\tau=3,2.25$, and 1.5, respectively, so that one could obtain three different response modes of amplitude-frequency curve shown in Figure 1, where the solid line is for the stable solution and the dot line is for the unstable one.

Next the numerical formula [22] is adopted as

$$
D^{p}\left[x\left(t_{l}\right)\right] \approx h^{-p} \sum_{j=0}^{l} C_{j}^{p} x\left(t_{l-j}\right)
$$

where $t_{l}=\operatorname{lh}(l=1,2,3, \ldots)$ is the time sample points, $h$ is step length, and $C_{j}^{p}$ is the fractional binomial coefficient with the iterative relationship as

$$
\begin{aligned}
C_{0}^{p} & =1, \\
C_{j}^{p} & =\left(1-\frac{1+p}{j}\right) C_{j-1}^{p} .
\end{aligned}
$$




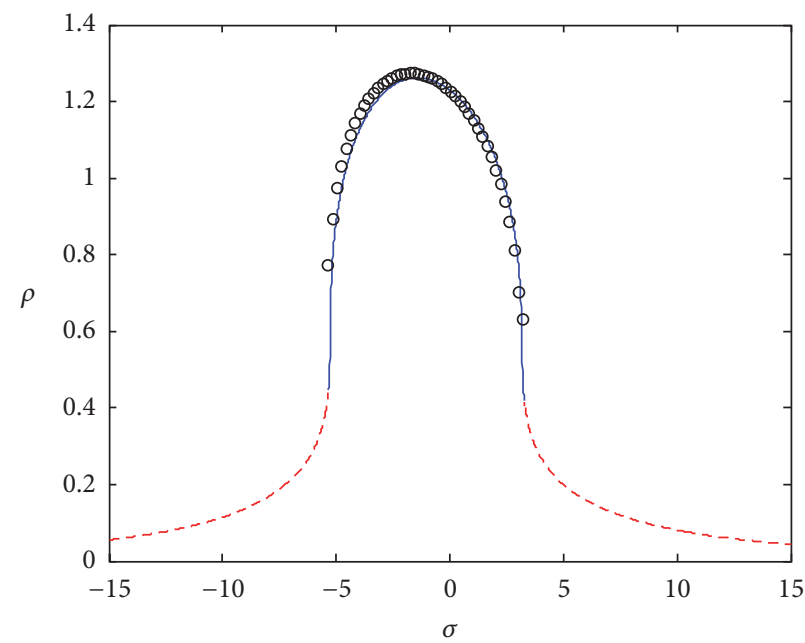

(a) $\tau=3$

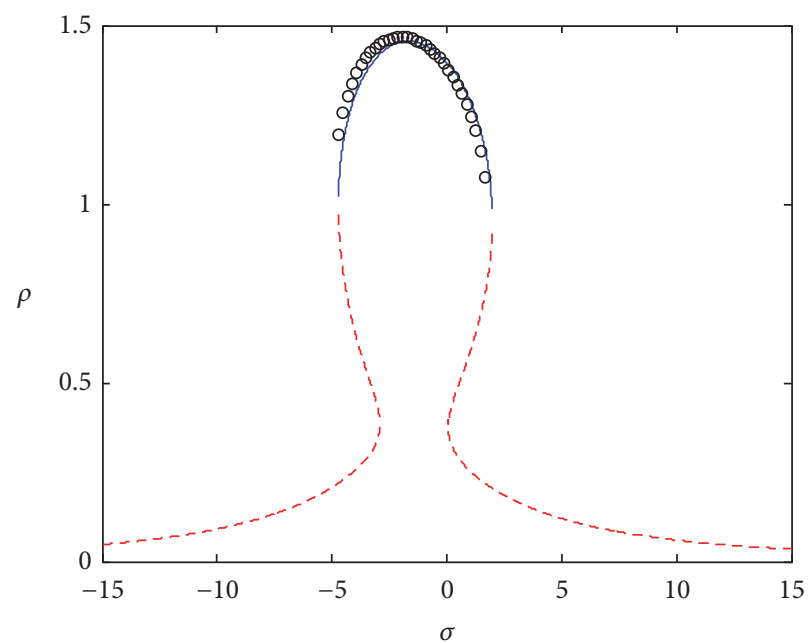

(b) $\tau=2.25$

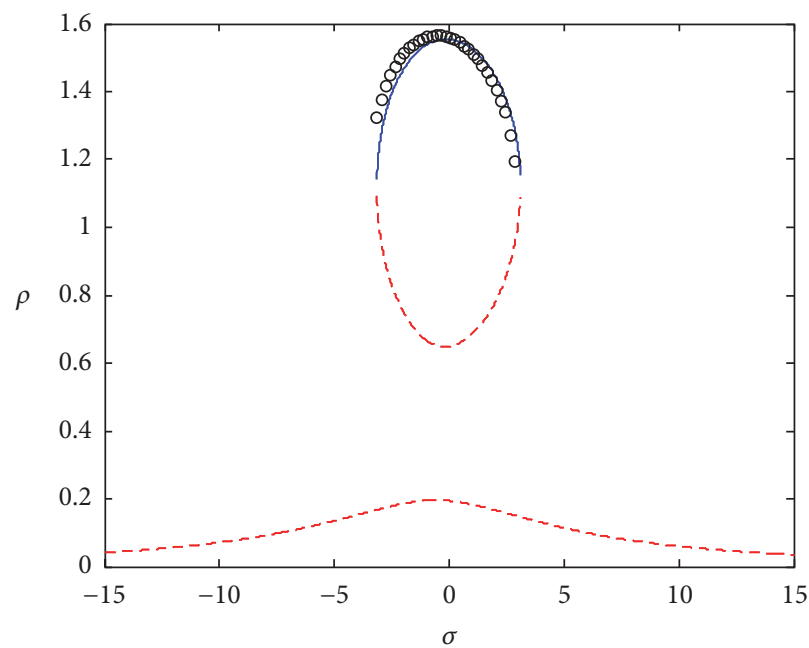

(c) $\tau=1.5$

FIGURE 1: Comparisons of the amplitude-frequency curves by the approximate analytical solution with that by numerical integration in three response modes.

Letting $\tau=i \times h$, where $i$ is natural number, one could obtain [37]

$$
D^{p}[x(t-\tau)]=D^{p}[x(t-i h)] .
$$

Based on (28)-(30), one could get the numerical iterative algorithm of (1) as follows:

$$
\begin{aligned}
& X_{1}\left(t_{l}\right)=X_{2}\left(t_{l-1}\right) h-\sum_{j=1}^{l} C_{j}^{1} X_{1}\left(t_{l-j}\right), \\
& X_{2}\left(t_{l}\right)=\frac{1}{m}\left\{K_{1} X_{3}\left(t_{l-1-i}\right)\right. \\
& \quad-\alpha_{1}\left[X_{1}^{2}\left(t_{l-1}\right)-1\right] X_{2}\left(t_{l-1}\right)-k X_{1}\left(t_{l-1}\right) \\
& \left.\quad+F \cos \left(\omega \cdot t_{l-1}\right)\right\} h-\sum_{j=1}^{l} C_{j}^{1} X_{2}\left(t_{l-j}\right),
\end{aligned}
$$

$$
X_{3}\left(t_{l}\right)=X_{2}\left(t_{l-1}\right) h^{1-p}-\sum_{j=1}^{l} C_{j}^{1-p} X_{3}\left(t_{l-j}\right),
$$

where $X_{1}=x(t)$ is displacement, $X_{2}=\dot{x}(t)$ is velocity, and $X_{3}=D^{p}[x(t)]$ is the fractional derivative of displacement. Here we select $h=0.001$, and the total computation time is generally $300 \mathrm{~s}$. Omitting the frontal $80 \%$ response, we take the maximum value of the posterior $20 \%$ response as the steady-state amplitude of the numerical results. As a comparison, the amplitude-frequency curve by numerical integration is also shown in Figure 1 denoted by small circles. From Figure 1, it could be found that the approximate analytical solutions agree very well with the numerical results and achieve satisfactory precision in all the three response modes.

4.2. Effects of the Fractional Parameters on the AmplitudeFrequency Curves. Now considering the system parameters $\alpha_{1}=1, m=4, k=10, p=0.5$, and $\tau=3$, one could obtain 


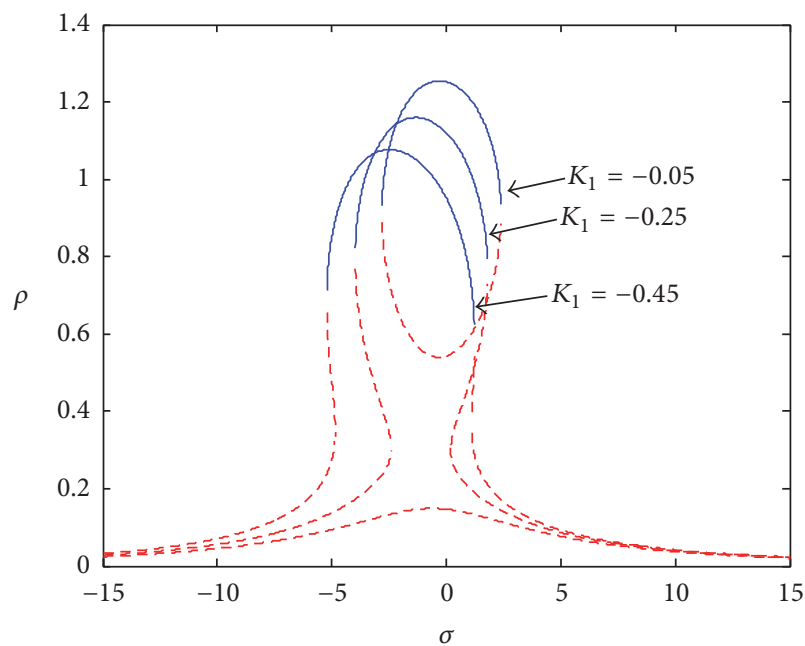

(a) $\tau=3 ; F=1$

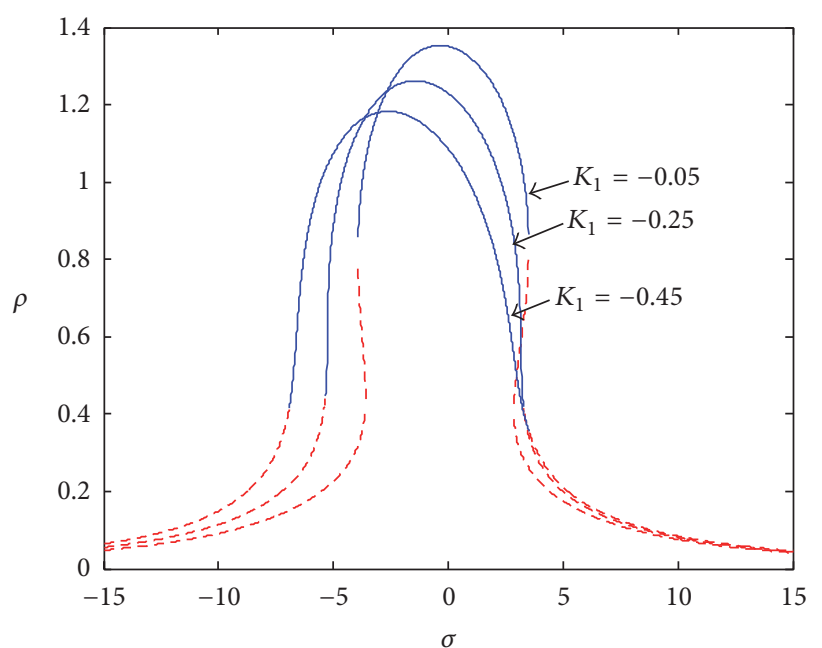

(b) $\tau=3 ; F=1.4$

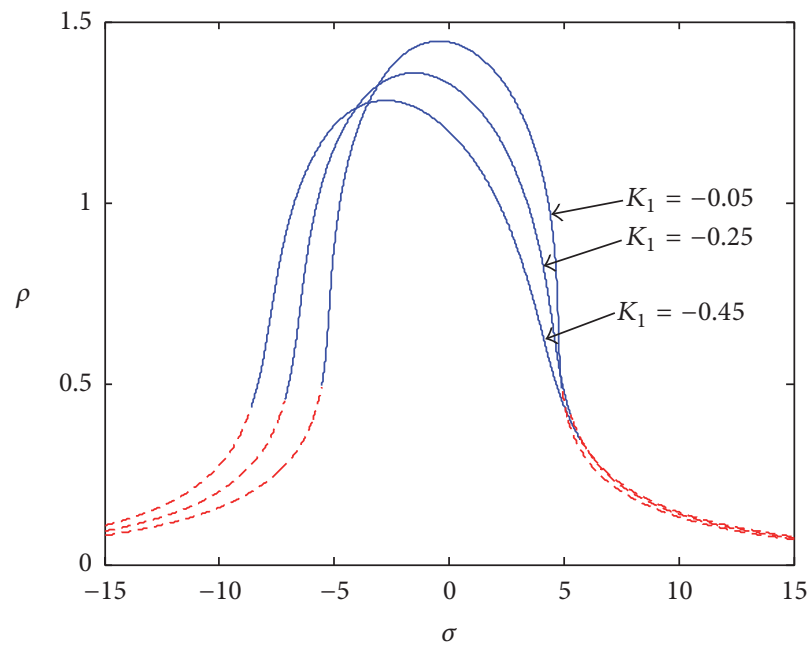

(c) $\tau=3 ; F=1.8$

FIGURE 2: Effects of the fractional feedback gain $K_{1}$ on the amplitude-frequency curves in three response modes $(p=0.5)$.

the amplitude-frequency curves shown in Figure 2 when the fractional feedback gain $K_{1}$ varies. From Figure 2, one could find that the response amplitude decreases when the fractional feedback gain $K_{1}$ decreases gradually, which means the equivalent damping of the system increases with the decrease of $K_{1}$. Moreover, the resonance frequency will increase along with the decrease of the fractional feedback gain $K_{1}$, which is because the equivalent stiffness coefficient becomes also larger. In this process, the amplitude-frequency curve of the system is shifted to the left. From Figure 2(a), one could find that the topology structure of amplitude-frequency curve is even changed due to the variation of $K_{1}$. It can be seen that the decrease of fractional-order feedback gain $K_{1}$ leads to the increase of the resonance frequency (i.e., natural frequency) of the system, and the amplitude-frequency curve is shown to be shifted and its topology structure is also changed.

Next we select the system parameters $\alpha_{1}=1, m=4$, $k=10, K_{1}=-0.25$, and $\tau=3$. When the fractionalorder $p$ changes, one could obtain the amplitude-frequency curves shown in Figure 3. It could be found that the larger the fractional-order $p$, the larger the maximum amplitude. The reason is that the equivalent linear damping coefficient will decrease along with the increase of fractional-order $p$. Moreover, the resonance frequency will be larger along with the increase of the fractional-order $p$, which is due to the fact that the equivalent stiffness coefficient becomes also larger. In this process, the amplitude-frequency curve of the system is shifted to the left. From Figure 3(a), it could be found that the topology structure of the amplitude-frequency curve will be changed due to the variation of $p$.

However, the above analysis is only applicable to the case of $\tau=3$. When time delay $\tau$ takes another value, the effects of the fractional-order $p$ on this system may vary, which is due to the fact that the fractional-order $p$ is coupling with time delay $\tau$ as trigonometric function in fractional-order delayed feedback.

Finally, the system parameters are selected as $\alpha_{1}=1$, $m=4, k=10, K_{1}=-0.25, p=0.5$, and $F=1.4$. The 


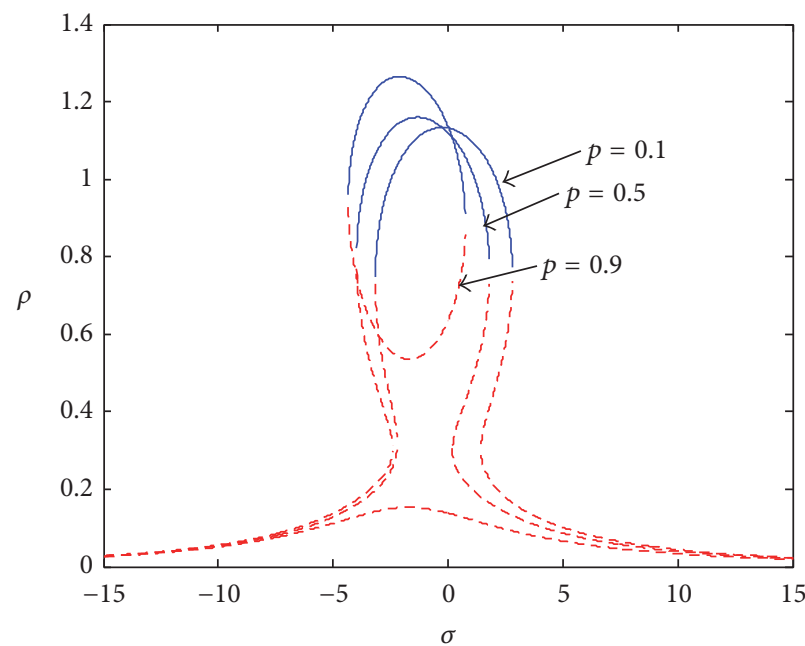

(a) $\tau=3 ; F=1$

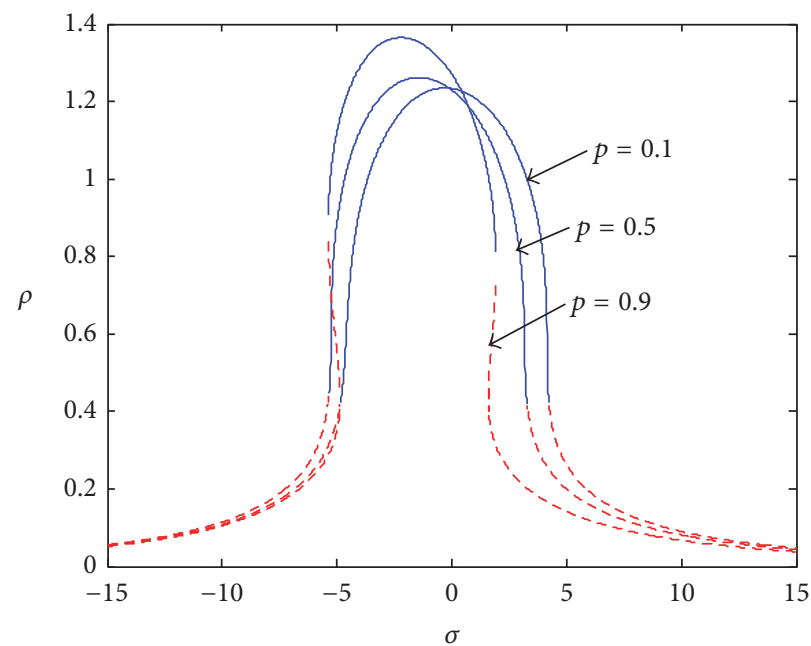

(b) $\tau=3 ; F=1.4$

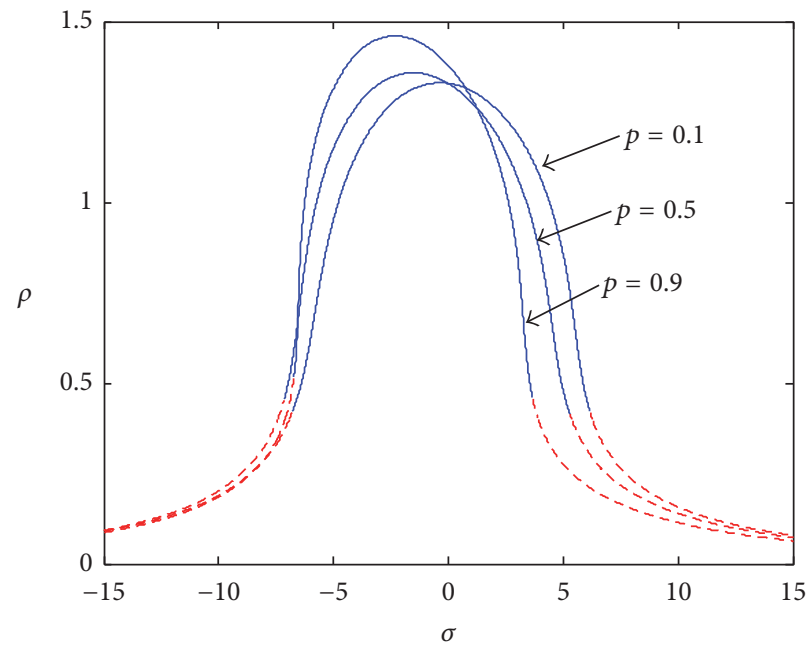

(c) $\tau=3 ; F=1.8$

FIGURE 3: Effects of the fractional-order $p$ on the amplitude-frequency curves in three response modes $\left(K_{1}=-0.25\right)$.

amplitude-frequency curves are shown in Figure 4 when time delay $\tau$ takes some different values. From the observation of Figure 4(a), one could find that the response amplitude of the system increases gradually when time delay $\tau$ is increased from 0.5. At the same time, the system resonance frequency decreases, the amplitude-frequency curve of the system shifts from right to left, and its topology structure changes. From Figure 4(b), we could see that the response amplitude of the system begins to decrease gradually when time delay $\tau$ continues to increase. Moreover, the resonance frequency increases, and the system amplitude-frequency curve continues to shift to the left. As shown in Figure 4(c), it could be found that the response amplitude of the system begins to increase gradually again as time delay $\tau$ continues to increase. Meanwhile, the resonance frequency decreases and the amplitude-frequency curve of the system shifts to the right gradually. It is easy to see that the amplitudefrequency curve of Figure 4(d) varies with time delay $\tau$ as shown in Figure 4(a), which is due to the equivalent damping and equivalent stiffness contain trigonometric functions in (17a) and (17b). One could obtain $T \approx 2 \pi / \omega_{0}=3.9738$. Therefore, time delay $\tau$ periodically affects the amplitudefrequency curve of the system (see Figure 5).

\section{Conclusions}

The primary resonance of van der Pol oscillator under fractional-order delayed negative feedback and forced excitation by averaging method is studied, and the approximate analytical solution is obtained. The steady-state solutions and stability conditions are investigated. The effects of the fractional feedback, the fractional order, and time delay on the solution are analyzed, which are characterized by the equivalent damping coefficient and equivalent stiffness coefficient. Moreover, it is found that the changes of fractional-order delayed feedback parameters may change the amplitude and topology structure of the amplitude-frequency curve. These results have important influence on the dynamical behavior 


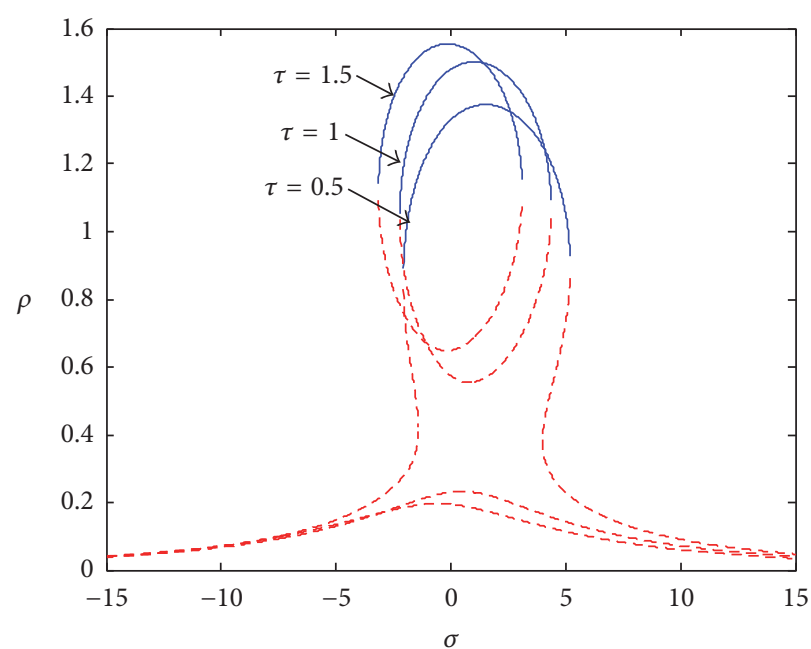

(a) $p=0.5 ; F=1.4$

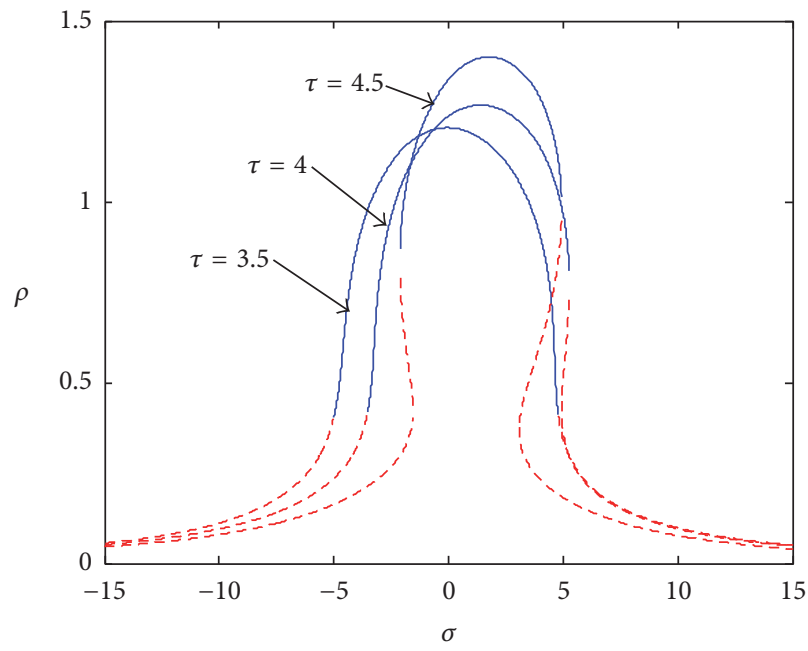

(c) $p=0.5 ; F=1.4$

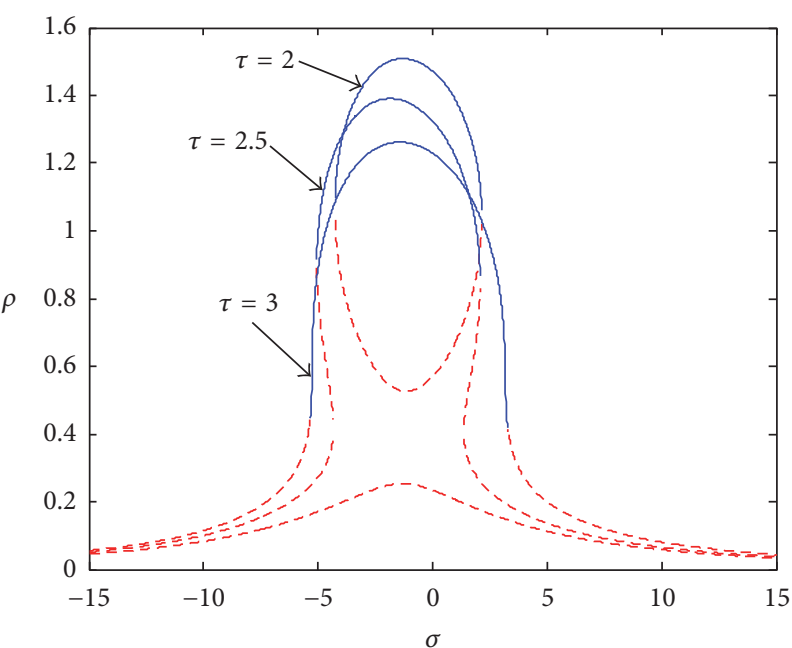

(b) $p=0.5 ; F=1.4$

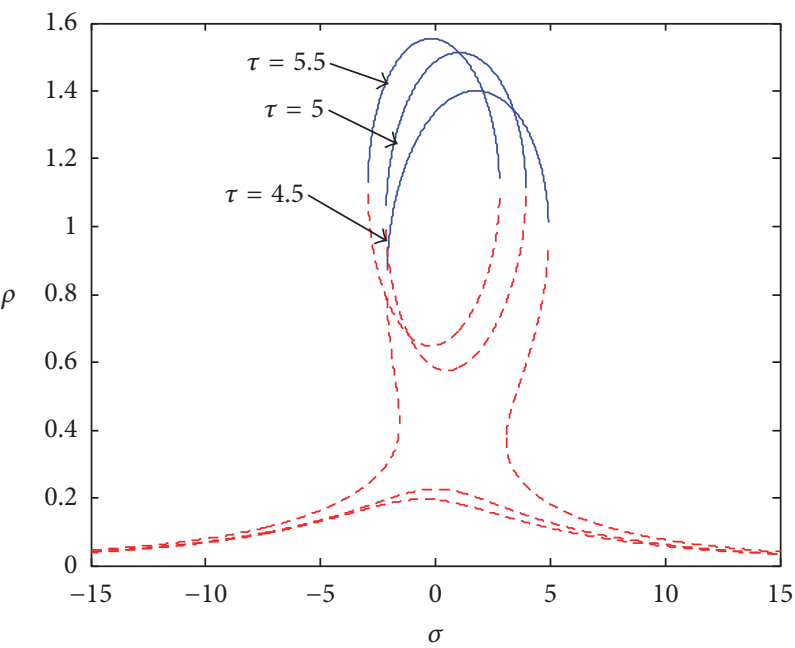

(d) $p=0.5 ; F=1.4$

FIGURE 4: Effects of the time delay $\tau$ on the amplitude-frequency curves $\left(K_{1}=-0.25\right)$.

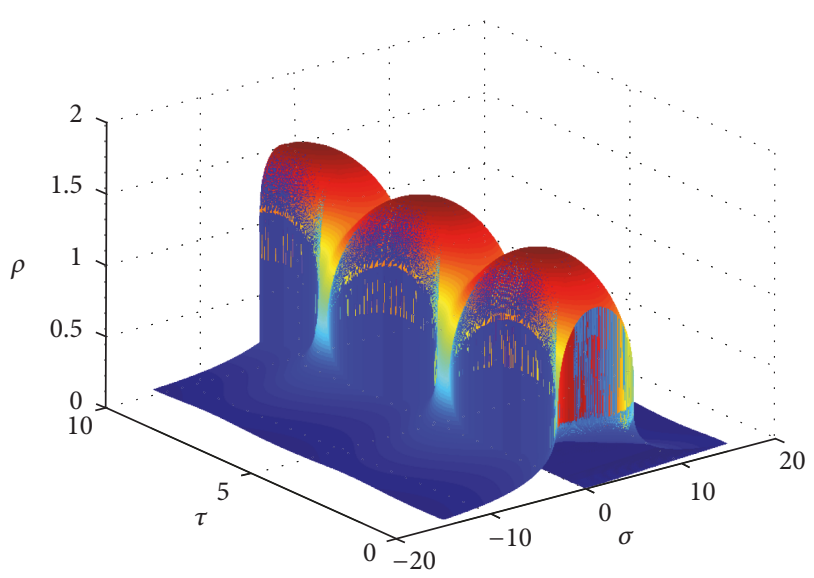

FIGURE 5: Relation between time delay, frequency difference, and amplitude.

and could be of great significance to the optimization and control of a similar system.

\section{Conflicts of Interest}

The authors declare that they have no conflicts of interest.

\section{Acknowledgments}

The authors are grateful to the support from the National Natural Science Foundation of China (no. 11672191), Hundred Talents Innovation Program of Hebei Province (SLRC2017053), and the Training Program for Leading Talent in University Innovative Research Team in Hebei Province (LJRC006).

\section{References}

[1] P. L. Butzer and U. Westphal, "An introduction to fractional calculus," in Applications of Fractional Calculus in Physics, pp. 1-85, World Scientific, Singapore, 2000.

[2] O. Heaviside, Electromagnetic Theory, Cambridge University Press, Cambridge, UK, 2011. 
[3] I. Podlubny, Fractional Differential Equations, vol. 198 of Mathematics in Science and Engineering, Academic Press, San Diego, Calif, USA, 1999.

[4] R. Hilfer, Applications of Fractional Calculus in Physics, World Scientific, Singapore, 2000.

[5] R. C. Koeller, "Applications of fractional calculus to the theory of viscoelasticity," American Society of Mechanical Engineers. Transactions of the ASME. Journal of Applied Mechanics, vol. 51, no. 2, pp. 299-307, 1984.

[6] N. Laskin, "Fractional market dynamics," Physica A: Statistical Mechanics and its Applications, vol. 287, no. 3-4, pp. 482-492, 2000.

[7] B. N. Lundstrom, M. H. Higgs, W. J. Spain, and A. L. Fairhall, "Fractional differentiation by neocortical pyramidal neurons," Nature Neuroscience, vol. 11, no. 11, pp. 1335-1342, 2008.

[8] C. Li, A. Chen, and J. Ye, "Numerical approaches to fractional calculus and fractional ordinary differential equation," Journal of Computational Physics, vol. 230, no. 9, pp. 3352-3368, 2011.

[9] A. Babakhani, D. Baleanu, and R. Khanbabaie, "Hopf bifurcation for a class of fractional differential equations with delay," Nonlinear Dynamics, vol. 69, no. 3, pp. 721-729, 2012.

[10] C. Li and Y. Ma, "Fractional dynamical system and its linearization theorem," Nonlinear Dynamics, vol. 71, no. 4, pp. 621-633, 2013.

[11] C. Huang, J. Cao, and M. Xiao, "Hybrid control on bifurcation for a delayed fractional gene regulatory network," Chaos, Solitons \& Fractals, vol. 87, pp. 19-29, 2016.

[12] Y. J. Shen, S. P. Yang, and H. J. Xing, "Dynamical analysis of linear single degree-of-freedom oscillator with fractional-order derivative," Acta Physica Sinica, vol. 61, no. 11, Article ID 110505, pp. 1-6, 2012.

[13] Y. Shen, S. Yang, H. Xing, and G. Gao, "Primary resonance of Duffing oscillator with fractional-order derivative," Communications in Nonlinear Science and Numerical Simulation, vol. 17, no. 7, pp. 3092-3100, 2012.

[14] Y. Shen, S. Yang, H. Xing, and H. Ma, "Primary resonance of Duffing oscillator with two kinds of fractional-order derivatives," International Journal of Non-Linear Mechanics, vol. 47, no. 9, pp. 975-983, 2012.

[15] Y. J. Shen, P. Wei, and S. P. Yang, "Primary resonance of fractional-order van der Pol oscillator," Nonlinear Dynamics, vol. 77, pp. 1629-1642, 2014

[16] Y. J. Shen, S. F. Wen, X. H. Li, S. P. Yang, and H. J. Xing, "Dynamical analysis of fractional-order nonlinear oscillator by incremental harmonic balance method," Nonlinear Dynamics, vol. 85, no. 3, pp. 1457-1467, 2016.

[17] L. Chen, W. Wang, Z. Li, and W. Zhu, "Stationary response of Duffing oscillator with hardening stiffness and fractional derivative," International Journal of Non-Linear Mechanics, vol. 48, pp. 44-50, 2013.

[18] L. C. Chen and W. Q. Zhu, "Stochastic response of fractionalorder van der Pol oscillator," Theoretical Applied Mechanics Letters, vol. 4, no. 1, Article ID 013010, 2014.

[19] Y. Yang, W. Xu, W. Jia, and Q. Han, "Stationary response of nonlinear system with Caputo-type fractional derivative damping under Gaussian white noise excitation," Nonlinear Dynamics, vol. 79, pp. 139-146, 2014.

[20] Y. G. Yang, W. Xu, X. D. Gu, and Y. H. Sun, "Stochastic response of a class of self-excited systems with Caputo-type fractional derivative driven by Gaussian white noise," Chaos, Solitons \& Fractals, vol. 77, pp. 190-204, 2015.
[21] Y. Xu, Y. Li, and D. Liu, "A method to stochastic dynamical systems with strong nonlinearity and fractional damping," Nonlinear Dynamics, vol. 83, no. 4, pp. 2311-2321, 2016.

[22] I. Petras, Fractional-Order Nonlinear System, Higher Education Press, China, 2011.

[23] C. Li and G. Peng, "Chaos in Chen's system with a fractional order," Chaos, Solitons \& Fractals, vol. 22, no. 2, pp. 443-450, 2004.

[24] E. Ahmed, A. M. El-Sayed, and H. A. El-Saka, "On some RouthHurwitz conditions for fractional order differential equations and their applications in Lorenz, Rössler, Chua and Chen systems," Physics Letters A, vol. 358, no. 1, pp. 1-4, 2006.

[25] X. Li and R. Wu, "Hopf bifurcation analysis of a new commensurate fractional-order hyperchaotic system," Nonlinear Dynamics, vol. 78, no. 1, pp. 279-288, 2014.

[26] J. Čermák and L. Nechvátal, "The Routh-Hurwitz conditions of fractional type in stability analysis of the Lorenz dynamical system," Nonlinear Dynamics, vol. 87, no. 2, pp. 939-954, 2017.

[27] H. Y. Hu and Z. H. Wang, Dynamics of controlled mechanical systems with delayed feedback, Springer Berlin Heidelberg, 2002.

[28] Z. H. Wang and H. Y. Hu, "Stability switches of time-delayed dynamic systems with unknown parameters," Journal of Sound and Vibration, vol. 233, no. 2, pp. 215-233, 2000.

[29] H. Wang and H. Hu, "Remarks on the perturbation methods in solving the second-order delay differential equations," Nonlinear Dynamics, vol. 33, no. 4, pp. 379-398, 2003.

[30] W. Deng, C. Li, and J. Lü, "Stability analysis of linear fractional differential system with multiple time delays," Nonlinear Dynamics, vol. 48, no. 4, pp. 409-416, 2007.

[31] M. Shi and Z. Wang, "An effective analytical criterion for stability testing of fractional-delay systems," Automatica, vol. 47, no. 9, pp. 2001-2005, 2011.

[32] Z. Guo, A. Y. T. Leung, and H. X. Yang, "Oscillatory region and asymptotic solution of fractional van der Pol oscillator via residue harmonic balance technique," Applied Mathematical Modelling, vol. 35, no. 8, pp. 3918-3925, 2011.

[33] A. Y. T. Leung, H. X. Yang, and Z. J. Guo, "The residue harmonic balance for fractional order van der Pol like oscillators," Journal of Sound and Vibration, vol. 331, no. 5, pp. 1115-1126, 2012.

[34] Q. X. Liu, J. K. Liu, and Y. M. Chen, "Asymptotic limit cycle of fractional van der Pol oscillator by homotopy analysis method and memory-free principle," Applied Mathematical Modelling, vol. 40, no. 4, pp. 3211-3220, 2016.

[35] F. Xie and X. Lin, "Asymptotic solution of the van der Pol oscillator with small fractional damping," in Proceedings of the 3rd International Workshop on Fractional Differentiation and Its Applications, FDA08, vol. 136, pp. 1-4, Turkey, October 2009.

[36] Y. J. Shen, S. P. Yang, and C. Y. Sui, "Analysis on limit cycle of fractional-order van der Pol oscillator," Chaos, Solitons \& Fractals, vol. 67, pp. 94-102, 2014.

[37] S. F. Wen, Y. J. Shen, S. P. Yang, and J. Wang, "Dynamical response of Mathieu-Duffing oscillator with fractional-order delayed feedback," Chaos, Solitons \& Fractals, vol. 94, pp. 5462, 2017. 


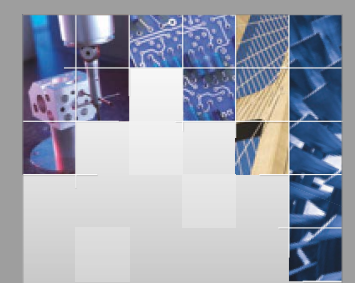

\section{Enfincering}
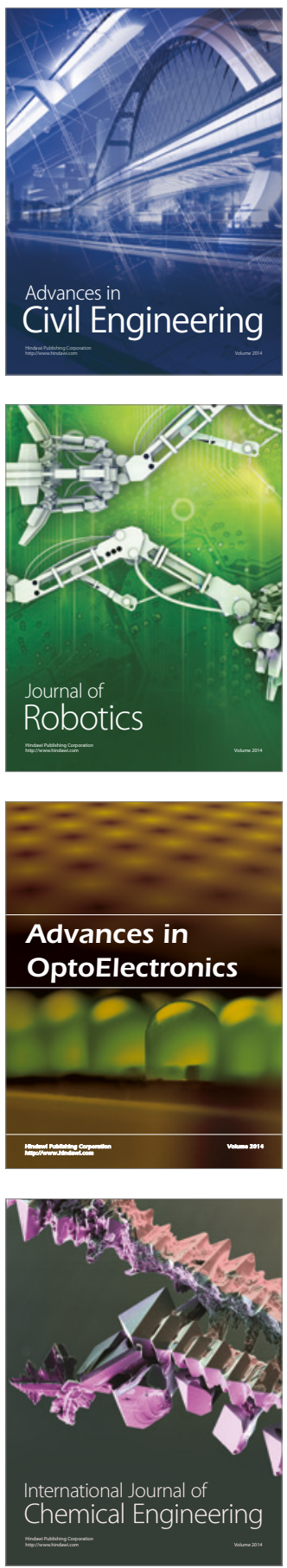

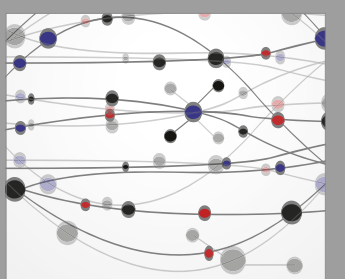

The Scientific World Journal

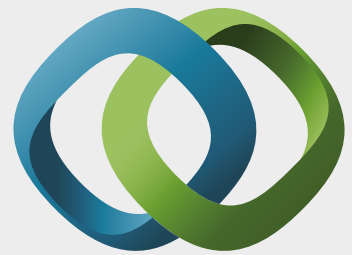

\section{Hindawi}

Submit your manuscripts at

https://www.hindawi.com
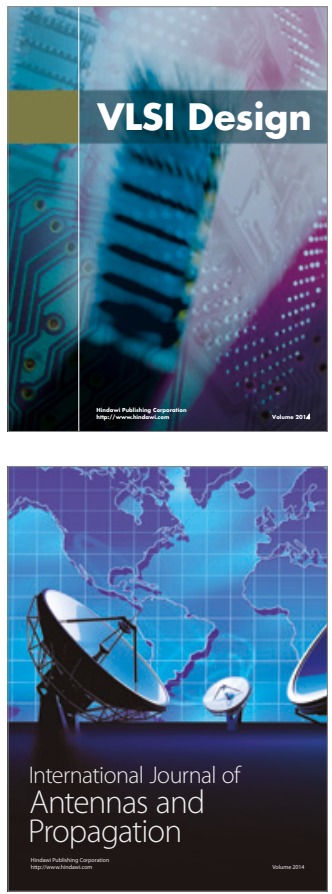

\section{Rotating}

Machinery
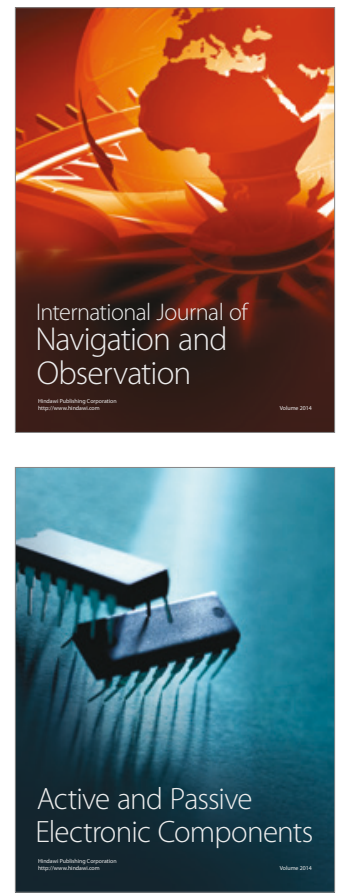
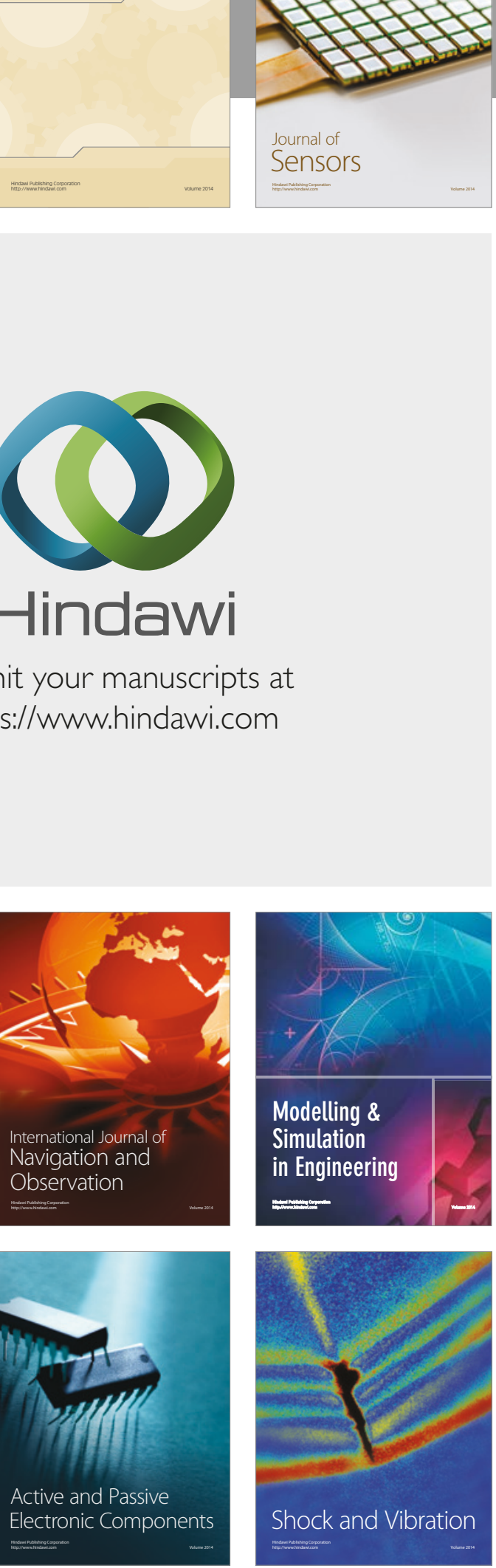
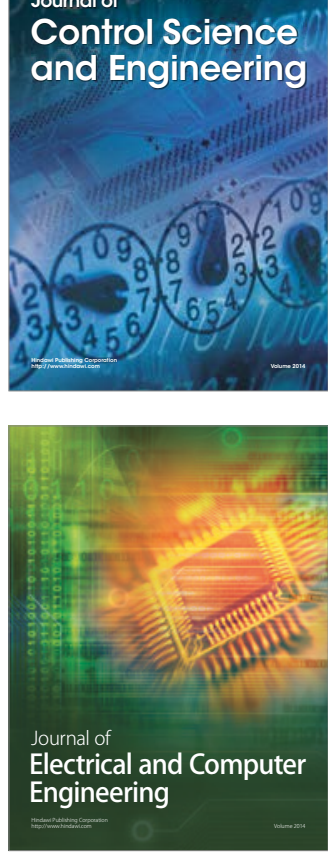

Distributed

Journal of

Control Science

and Engineering
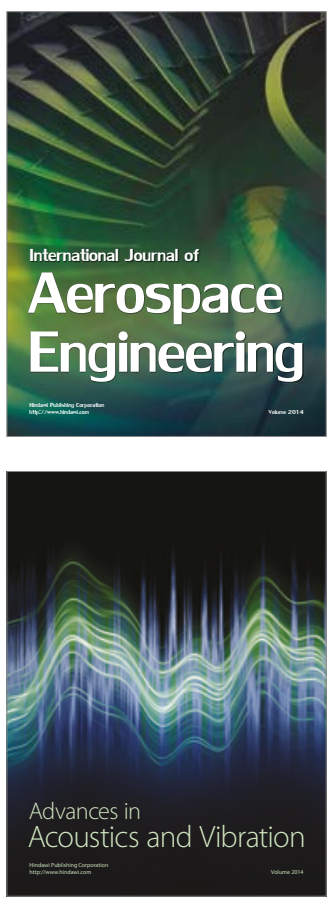

Sensor Networks 